\title{
Nonlinear Dynamic Modeling and Simulation of Circular Crane Anti-sway Payload
}

\author{
Xiangdong Li ${ }^{1,}$, , Wei Zhang ${ }^{1, b}$, Xindong Zhang ${ }^{1, c}$ and Qiangshuai Lin ${ }^{1, d}$ \\ ${ }^{1}$ Special Equipment Safety Supervision and Inspection Institute of Jiangsu Province, China \\ alxd1963@163.com, brebol123@163.com, ${ }^{\mathrm{a}} 22729257 @ q q . c o m,{ }^{d}$ lqsh2003@163.com
}

Keywords: Circular crane, Anti-sway control, Dynamic simulation

Abstract. Based on the analysis of the circular crane working principle, nonlinear dynamic mathematical model is established for the anti-sway payload, and the dynamic simulation is carried out by using Adams. It is verified that the swing angle could be reduced by controlling the acceleration of trolley and cart separately.

\section{Introduction}

Circular crane is installed in the nuclear power plants as important lifting transport equipment, which needs a high precision positioning and safety requirement. Although some enterprises in China have the ability of design and manufacture such as Taiyuan heavy industry and Dalian heavy industry, many circular cranes are imported ${ }^{[1]}$.

Generally, there are two main methods, which includes active anti sway method and passive anti sway method. Specifically, it includes mechanical type, hydraulic oil cylinder type, steel wire rope type, mechanical and electronic type, and intelligent electronic type ${ }^{[2]}$. Compared with the mechanical control system and hydraulic control system, intelligent electronic anti sway system has more advantages such as less additional equipment and weight, short swing time, and anti-swing control simultaneously.

In the process of research at the Tianwan nuclear power station which is built in Lianyungang city, sometimes the circular crane was not used with a very slow speed by workers in the nuclear internal for the sake of safety, because the payload swing is concerned with the trolley speed and trolley acceleration. Accurately, slow speed method for anti sway is effective but inefficient ${ }^{[3]}$. There is no literature reported in public that the anti sway control as a key technology had been used in the circular crane payload.

\section{Working principle of the circular crane}

Composition of the circular crane and relative parameters

The circular crane is installed inside the reactor building, which consists of several main parts as follows.

a. the bridge and the rotating mechanism

b. the trolley set on the main lifting mechanism of 217 tons and the deputy lifting mechanism of 10 tons.

c. the installation trolley set on lifting mechanism of 190 tons. The hook is connected with the main hook of 217 tons though the trunnion of joint lifting beam, which could get the 396 tons lifting capacity. There is only one installation trolley shared with two circular crane, which will be removed from the circular crane after the two reactors equipment installation.

d. the mobile crane of 5 tons which can move through two directions. It consists of the main girder floor of fixed guide rail, walking beam and service trolley that can take 5 tons weight.

e. the central arch support across in the bridge above for supporting the pulley of 60 tons block and guide pulleys. 

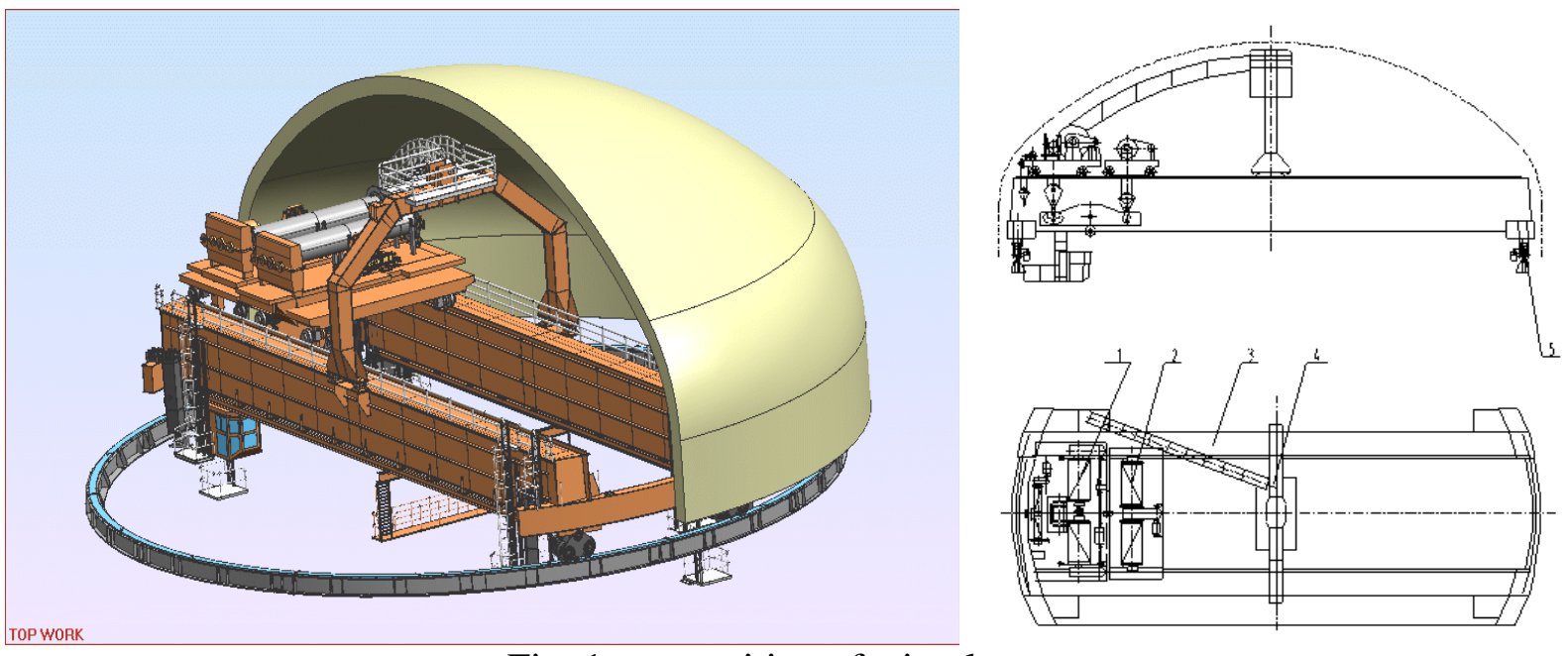

Fig. 1 composition of circular crane

Table 1 shows the main kinetic parameters of circular crane

Table 1 the main kinetic parameters

\begin{tabular}{ccccc}
\hline & $\begin{array}{c}\text { Lifting range } \\
{[\mathbf{m}]}\end{array}$ & $\begin{array}{c}\text { Lifting speed } \\
{[\mathbf{m} / \mathbf{m i n}]}\end{array}$ & $\begin{array}{c}\text { Running speed } \\
{[\mathbf{m} / \mathbf{m i n}]}\end{array}$ & $\begin{array}{c}\text { Rotational speed } \\
{[\mathbf{m} / \mathbf{m i n}]}\end{array}$ \\
\hline trolley & 42 & $0 \sim 10$ & $0 \sim 10$ & $/$ \\
cart & $/$ & $/$ & $/$ & $0 \sim 20$ \\
\hline
\end{tabular}

Note: It is allowed that the lifting, running and rotation can proceed simultaneously.

The factors of swing

The circular crane is different from the general crane that the cart of circular crane runs rotationally while the cart of others runs along a straight line. Just because of the rotational motion of cart, it is much more complicated that the cart rotation could generate centrifugal force whose component force's direction is synthetic or reverse to trolley's. In addition, the cart rotation and trolley linear motion influence each other, and that causes the Coriolis acceleration whose direction is determined by the rotational direction and linear direction.

According to the above analysis, the payload swing is concerned with cart velocity, cart angular acceleration and trolley acceleration. It should be noted that the steel wire rope length is one factor of swing, which is not taken into account in this paper. By the method of electronic anti sway, the motors of the cart and trolley should be controlled.

\section{Dynamic modeling of circular crane}

The radius of fixed pulley is assumed as zero and simplified to a point. In addition, the friction and air damping are ignored for convenience. The trolley $m_{1}$ and payload $m_{2}$ are connected by steel wire rope whose length is $l$. The angle between payload and plumb line is $\theta . \theta_{r}$ and $\theta_{\varphi}$ are the two components of $\theta$ in plane $x o z$ and plane $x o y$ which is in the moving coordinate system oxyz. The trolley driving force along the axis $x$ is $F_{r}$ and the cart driving force around the axis $z$ is $F_{\varphi}$. The Fig. 2 shows the coordinate system of circular crane. 


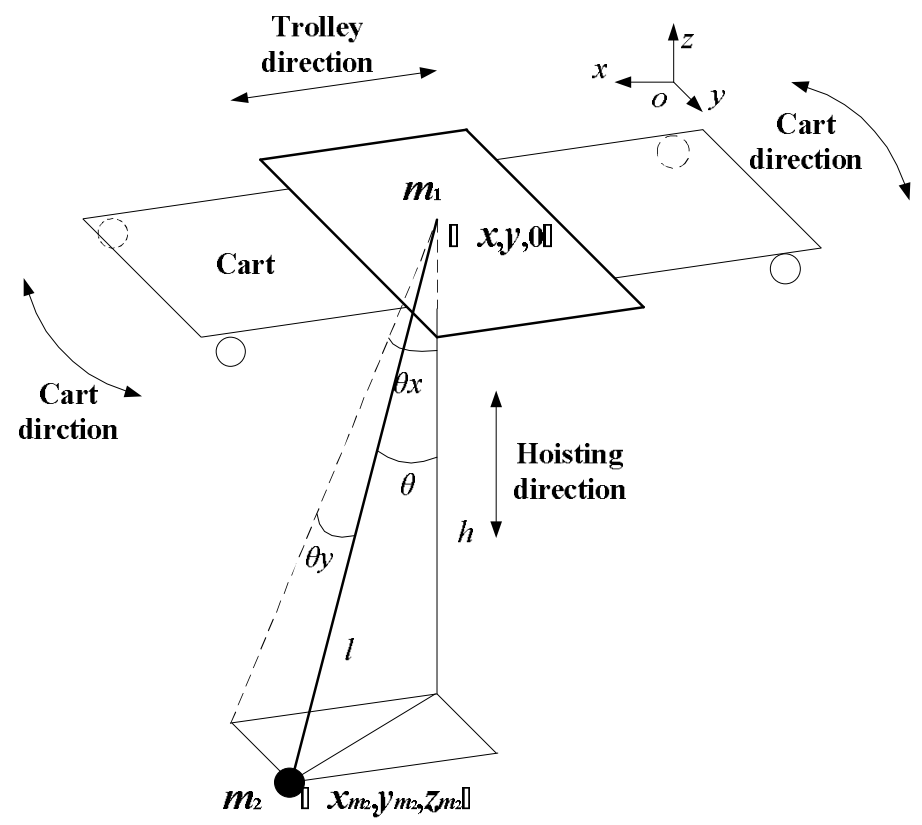

Fig. 2 coordinate system of circular crane

Then the trolley location is $(r, \varphi, 0)$ and the payload location is $\left(r_{2}, \varphi_{2}, z_{2}\right)$. Eq. 1 shows the relationship between trolley location and payload location.

$$
\left\{\begin{array}{l}
r_{2}=r+l \sin \theta_{r} \cos \theta_{\varphi} \\
\varphi_{2}=\varphi+\theta_{\varphi} \\
z_{2}=-l \cos \theta_{r} \cos \theta_{\varphi}
\end{array}\right.
$$

The velocity components of payload along $r, \varphi$ and $z$ is showed in Eq. 2.

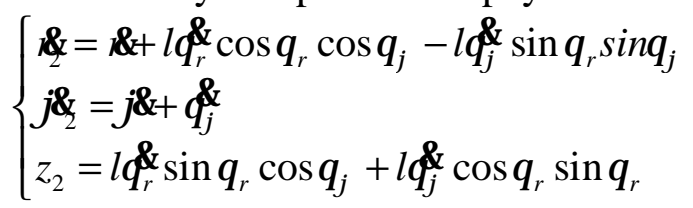

The kinetic energy of system:

$$
T=\frac{1}{2} m_{1}\left(\&+r^{2} \phi \delta\right)+\frac{1}{2} m_{2}\left[\left(\&+l \theta_{r}^{\alpha}-l \phi_{\varphi}^{\delta} \theta_{r} \theta_{\varphi}\right)^{2}+r^{2}\left(\phi \&+\theta_{\varphi}^{\alpha}\right)^{2}+\left(l \theta_{r}^{\delta} \theta_{r}+l \theta_{\varphi}^{\delta} \theta_{\varphi}\right)^{2}\right]
$$

The point $o_{l}$ hanging on the trolley is selected as a point of zero potential energy, then the system potential energy is

$$
V=-m_{2} g l
$$

Generalized coordinates is $\left(r, \varphi, l, \theta_{\mathrm{r}}, \theta_{\varphi}\right)$, and the Lagrange's equation is

$$
\frac{d}{d t}\left(\frac{\partial L}{\partial \phi_{i}}\right)-\frac{\partial L}{\partial q_{i}}=F_{q_{i}} \quad(i=1,2, \mathrm{~L}, 5)
$$

In Eq. 5, Lagrange function $L=T-V$, generalized coordinate $q_{i}$ are $x, y, l, \theta_{r}$ and $\theta_{\varphi}$, generalized force $F_{q_{i}}$ are $f_{r}, f_{\varphi}, f_{l}, 0$ and 0 .

According to Eq. 1 to Eq. 4, parameter $x, y, l, \theta_{r}$ and $\theta_{\varphi}$ are putted into Eq. 5, thus we get five equations. Practically, considering of the safety of payload transportation, the pendulum angle of payload is small. It could be simplified linearly as follows.

$$
\left\{\begin{array}{l}
\sin \theta_{r} \approx \theta_{r} \sin \theta_{\varphi} \approx \theta_{\varphi} \\
\cos \theta_{r} \approx 1, \cos \theta_{\varphi} \approx 1 \\
\sin \theta_{r} \sin \theta_{\varphi} \approx 0, \cos \theta_{r} \cos \theta_{\varphi} \approx 1
\end{array}\right.
$$

Thus, the five equations could be simplified as Eq. 6 . 


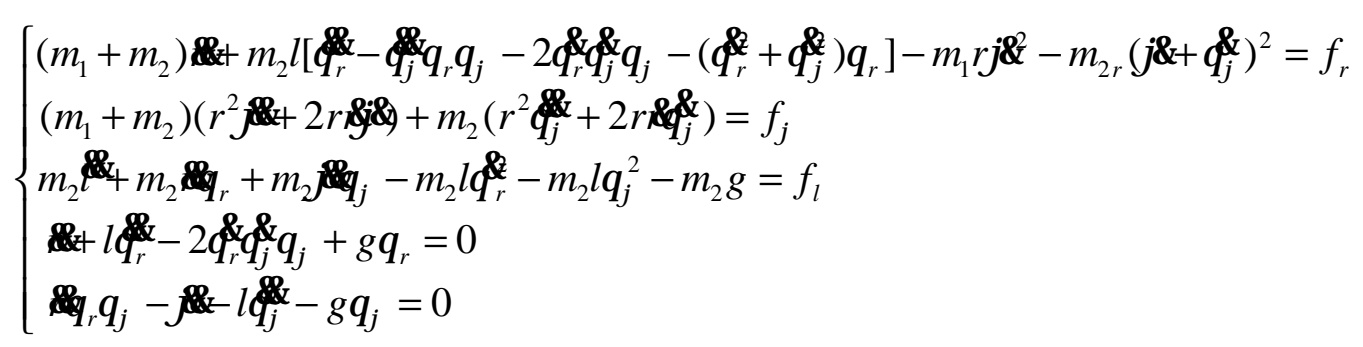

It indicated that the angle $\theta_{r}$ and $\theta_{\varphi}$ is determined by the acceleration and velocity of trolley and cart, unless the steel wire rope length is another factor.

\section{Dynamic simulation of circular crane}

We build the dynamic model in Adams. The bridge span is $42 \mathrm{~m}$ which makes circular motion around its centre of mass. The steel wire rope length is $10 \mathrm{~m}$.

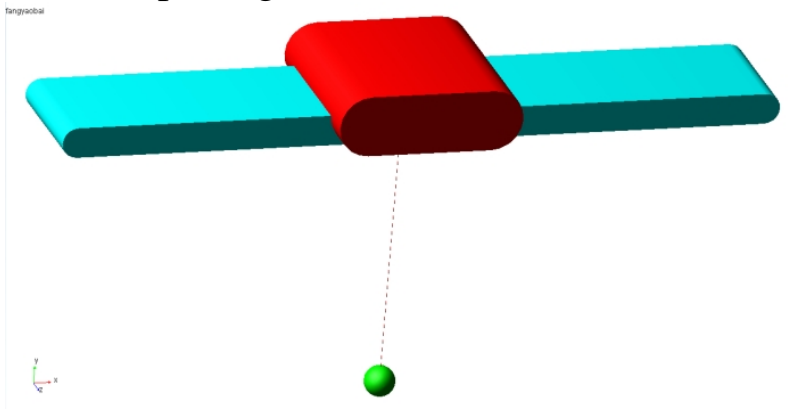

Fig. 3 Adams model of anti-sway payload

According to the simple pendulum cycle formula $T=2 \pi \sqrt{l / g}$, the period is $6.396 \mathrm{~s}$.

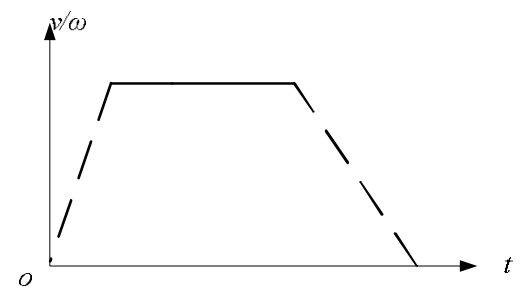

Fig. 4 loading process of velocity

If the trolley runs at a accelerated speeds of $0.2 \mathrm{~m} / \mathrm{s}^{2}, 0.4 \mathrm{~m} / \mathrm{s}^{2}, 0.6 \mathrm{~m} / \mathrm{s}^{2}$ and the accelerated time is 10s, the simulation result shows as Fig. 5. The curve includes acceleration motion and uniform motion. In the accelerated process, the payload swings like a simple pendulum but the symmetry axis is not the plumb line. There is energy change in the process of conversion between two motions.
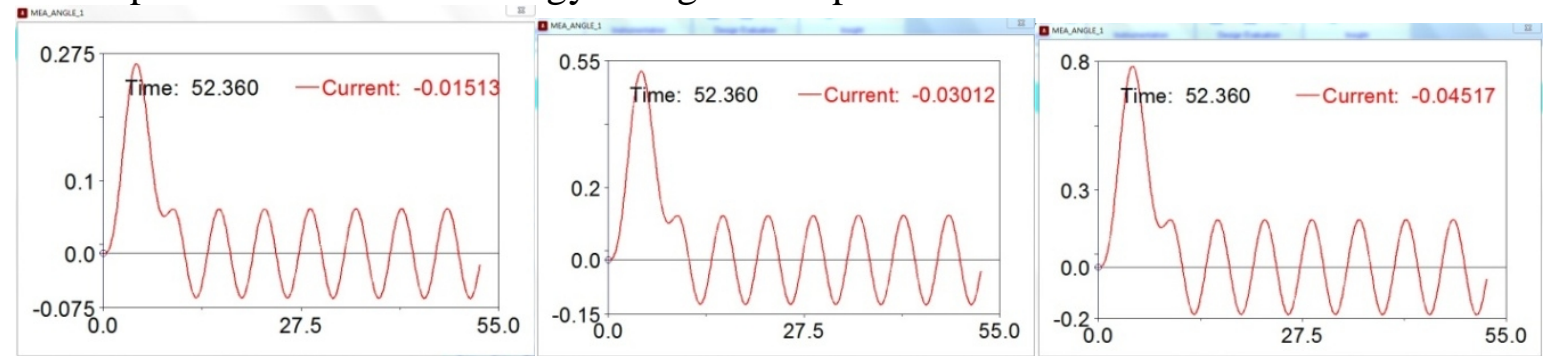

Fig. 5 simulation result of anti-sway process

Table 2 proportional relationship between acceleration and swing angle

\begin{tabular}{cc}
\hline Acceleration $\left[\mathbf{m} / \mathbf{s}^{2}\right]$ & Ultimate swing angle $\left[{ }^{\circ}\right]$ \\
\hline 0.2 & 0.05 \\
0.4 & 0.10 \\
0.6 & 0.15 \\
\hline
\end{tabular}


According to the table above, it could be conclude that when the angle is small, the swing angle is proportional to the trolley acceleration. Also, the process of conversion between two motions is a important factor which determines the last swing angle. Thus the position of payload process changing is what we consider about.

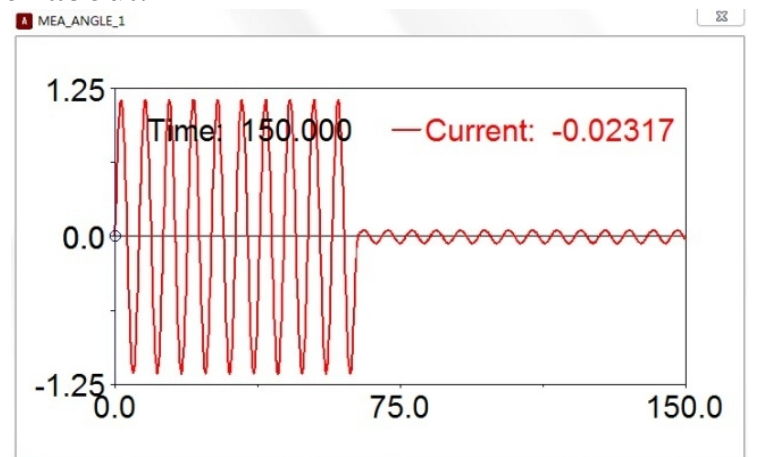

Fig. 6 optimized results of anti-sway payload

In the next simulation, the process changing time is set as $62.8 \mathrm{~s}$ around which is 10 times of swing period. At this very moment, the swing angle is about zero. Fig. 6 shows the obvious change of swing angle from $1.21^{\circ}$ to $0.03^{\circ}$. The simulation verified that the swing angle can be eliminated by an accelerated process easily.

\section{Conclusions}

The angle $\theta$ between rope and plumb line is determined by $\theta_{r}$ and $\theta_{\varphi}$ which are linear and orthogonal, so the angle could be reduced by controlling the acceleration of trolley and cart separately.

Take the trolley as an example, its deceleration position is the payload position where $\theta$ is zero, also the deceleration direction should be in the line with the payload velocity direction relative to trolley's. In the follow-up research work, the coupling relation between the payload position and the trolley velocity should be considered of.

\section{Acknowledgements}

This work was financially supported by the Jiangsu Province Quality and Technology Supervision Bureau (KJ112516).

\section{References}

[1] Wang Xiaojun, Shao Huihe. Fuzzy Logic-based Anti-Swing and Position Control for Bridge Cranes, Journal of System Simulation, Vol. 17 No. 4, Apr. 2005, p936-939.

[2] William Singhose, Lisa Porter, Michael Kenison, et. Effects of Payload on the Input Shaping Control of Gantry Cranes[J]. Control Engineering Practice, 2000, 8: p1159-1165.

[3] Mohamed B Trabia, Jamil M Renno, Kamal A F Moustafa. Generalized Design of an Anti-swing Fuzzy Logic Controller for an Overhead Crane with Hoist[J]. Journal of Vibration and Control, 2008, 14(3): p319-346.

[4] M B Bateman, I C Howard, A R Johnson, et. Computer Simulation of the Impact Performance of a Wire Rope Safety Fence[J]. International Journal of Impact Engineering, 2001,25: p67-85.

[5] Yang Jun Hua, Yang Kuang Shine. Adaptive Coupling Control for Overhead Crane Systems[Jl. Mechatronics, 2007, 17: p143-152.

[6] Andrew S E. Efficient Modeling of Extensible Cables and Pulley Systems in Adams[C]. London: Europe Adams Conference, 2002: p1-10.

[7] Etsujiro Imanishi, Takao Nanjo, Takahiro Kobayashi. Dynamic Simulation of Wire Rope With Contact[J]. Journal of Mechanical Science and Technology, 2009, 23: p1083-1088. 